\title{
A Baffling "Trilogy" --- Mediastinal Teratoma, Pulmonary Hamartoma and Hilar Granulomatous Lymphadenitis in a Single Patient: Unaccountable in Medical History
}

\author{
Authors \\ Meenu Gupta, Manisha Mahata, Sanghamitra Mukherjee, Tushar Kanti Das \\ Department of Pathology, R.G. Kar Medical College, Kolkata
}

\begin{abstract}
Mediastinal teratomas are the most common extra-gonadal germ cell tumours. They arise from ectopic pluripotent stem cells that fail to migrate from yolk endoderm to the gonad. They occur equally in men and women and usually are diagnosed in the second to fourth decade of life. Pulmonary hamartoma usually occurs as a benign, well-circumscribed single nodule in the lung parenchyma. Being more common in the sixth decade. Granulomatous lymphadenitis more commonly occurring in cervical lymph node is also commonly seen in hilar lymph node.

Here, we present a 17 year old male with all the three presentations, which itself is a rare occurrence.

Keyword: Mediastinal teratoma, pulmonary hamartoma, hilar Granuloma.
\end{abstract}

\section{INTRODUCTION}

Mature teratomas are the most common histological type of germ cell tumors. They manifest with a variety of clinical and radiological features. Pulmonary hamartoma $(\mathrm{PH})$ also known as mesenchymoma was first described in 1904 by Albrecht. In 1934, Goldsworthy applied this term to benign tumors located in the lung that were composed predominantly of a combination of fat and cartilage. Usually well-defined, solitary pulmonary nodules. It can occur in any part of the lungs, but are more often found in the periphery and rarely in the hilar parts.

Here, we present a case of a 17 year male with three unusual presentation, intrapulmonary teratoma, pulmonary hamartoma and granulomatous lymphadenitis.

\section{CASE REPORT}

A 17 year male, presented with a 4 month history of breathlessness. First, presented to a local health care where he was examined and decreased breath sounds on left side was noticed. A chest X-ray was done which showed pleural effusion and mass in the thoracic cavity on left side. Pleural fluid was drained out.

CT revealed a large thick walled multiloculated SOL with perifocal mass effect measuring $125 \times 82$ $\mathrm{mm}$ seen in the left hemithorax, anteriorly. The SOL is showing dense calcific foci, fat, fluid and soft tissue components.

Evidence of irregular thick wall air filled cavity measuring $35 \times 50 \times 65 \mathrm{~mm}$ seen in the left upper lobe with perifocal thickened septal lines.

Irregular area of alveolar collapse is seen in the left lower lobe. Few enlarged lymph nodes are 


\section{JMSCR Vol||05||Issue||07||Page 25805-25809||July}

seen in the right paratracheal and prevascularaor to pulmonary window region (Photomicrograph1).

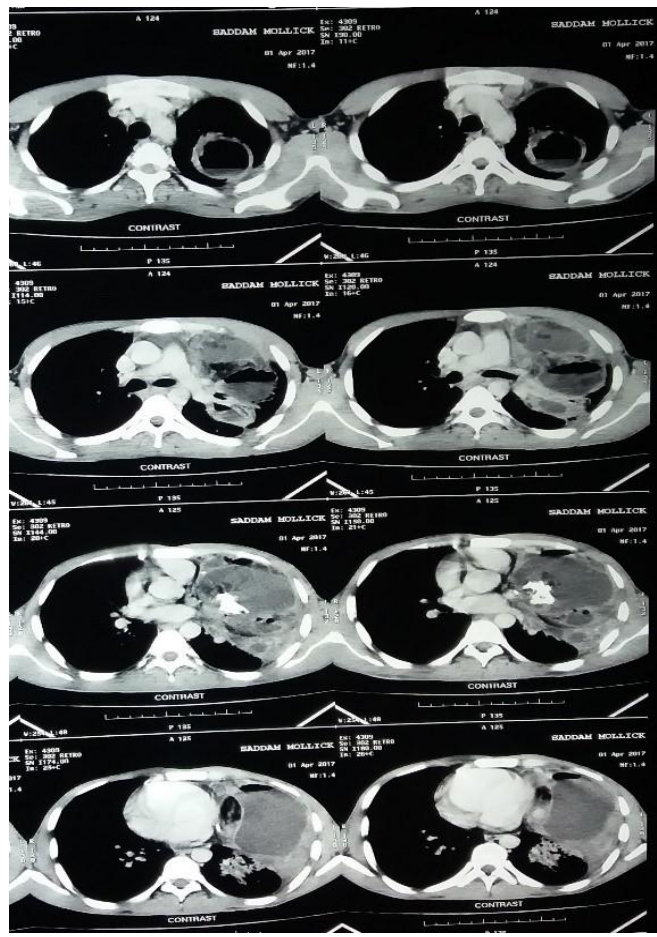

Photomicrograph 1: C T Scan revealing a multiloculated SOL in left hemithorax and an air filled cavity in left upper lobe.

Immediate surgical intervention was done and three specimens were sent to the department of pathology

Specimen 1: Single globular tissue piece measuring $10 \times 10 \times 5 \mathrm{~cm}$. Cut section is variegated, contains hair, bony structure and tooth. Microscopic examination showed a variety of cell lines consisting of squamous epithelium, hair follicles and sebaceous glands, cartilage and bony elements. Histopathological features are consistent with the diagnosis of mature teratoma. (Photomicrograph 2).

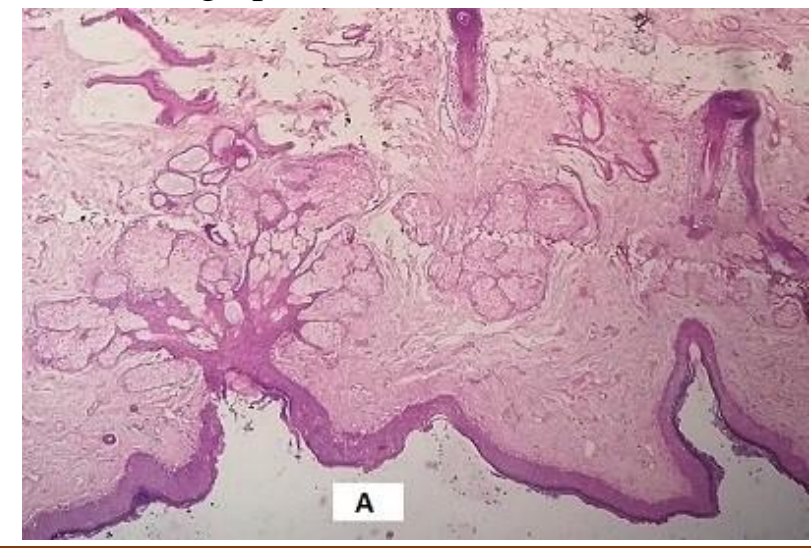

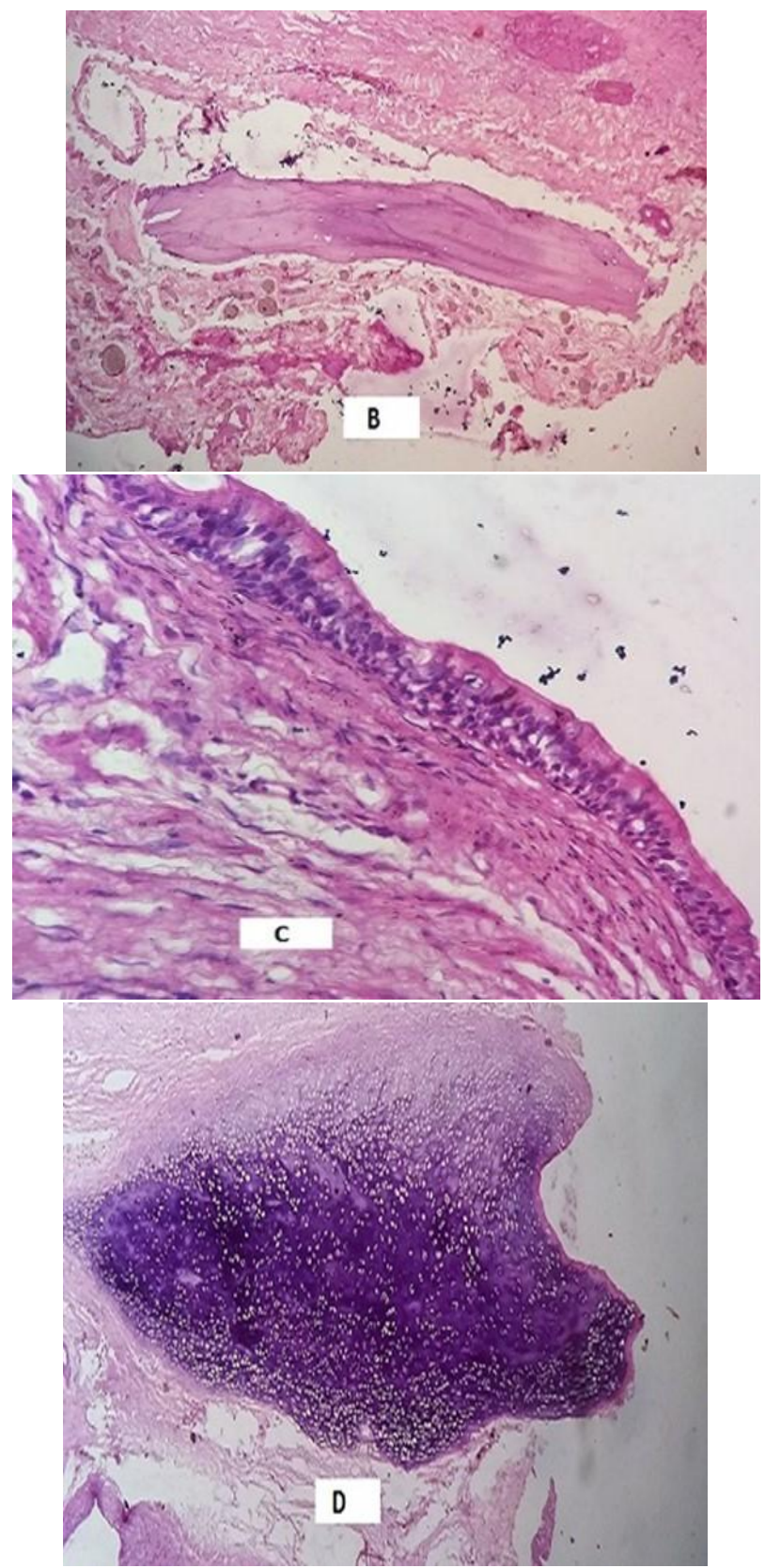

Photomicrograph 2 Microscopic examination showed a variety of mature cell lines - squamous epithelium and sebaceous glands (Fig. 2A), bone (Fig. 2B), pseudo stratified epithelium (Fig. 2C), cartilage (Fig. 2D)

Specimen 2: Single irregularly greyish brown tissue piece measuring $6.5 \times 5 \times 4.5 \mathrm{~cm}$. Cut section showed a cystic structure with some greyish brown and haemorrhagic area.

Microscopically-alobulocystic mass with fibro myxoid stroma admixed with areas showing alveoli and cartilage. Histopathological features are consistent with the diagnosis of Pulmonary hamartoma (Photomicrograph 3) 

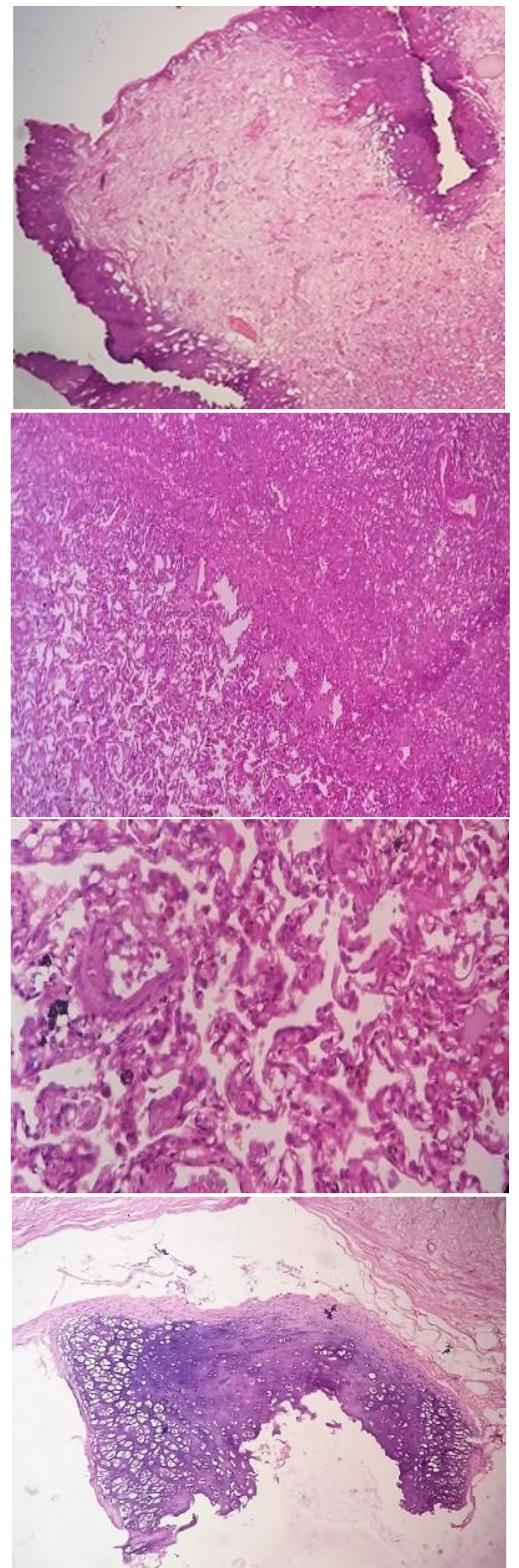

Photomicrograph 3: Microscopic examination showing alveoli and myxoid stroma with cartilage.
Specimen 3: Single, irregularly greyish brown tissue piece measuring $2 \mathrm{~cm}$ in maximum dimension.

Microscopical examination shows lymph nodal structure with areas of caseating necrosis and Langhans type of giant cells. Histopathological features are consistent with the diagnosis of caseating granulomatous lymphadenitis.

(Photomicrograph 4)

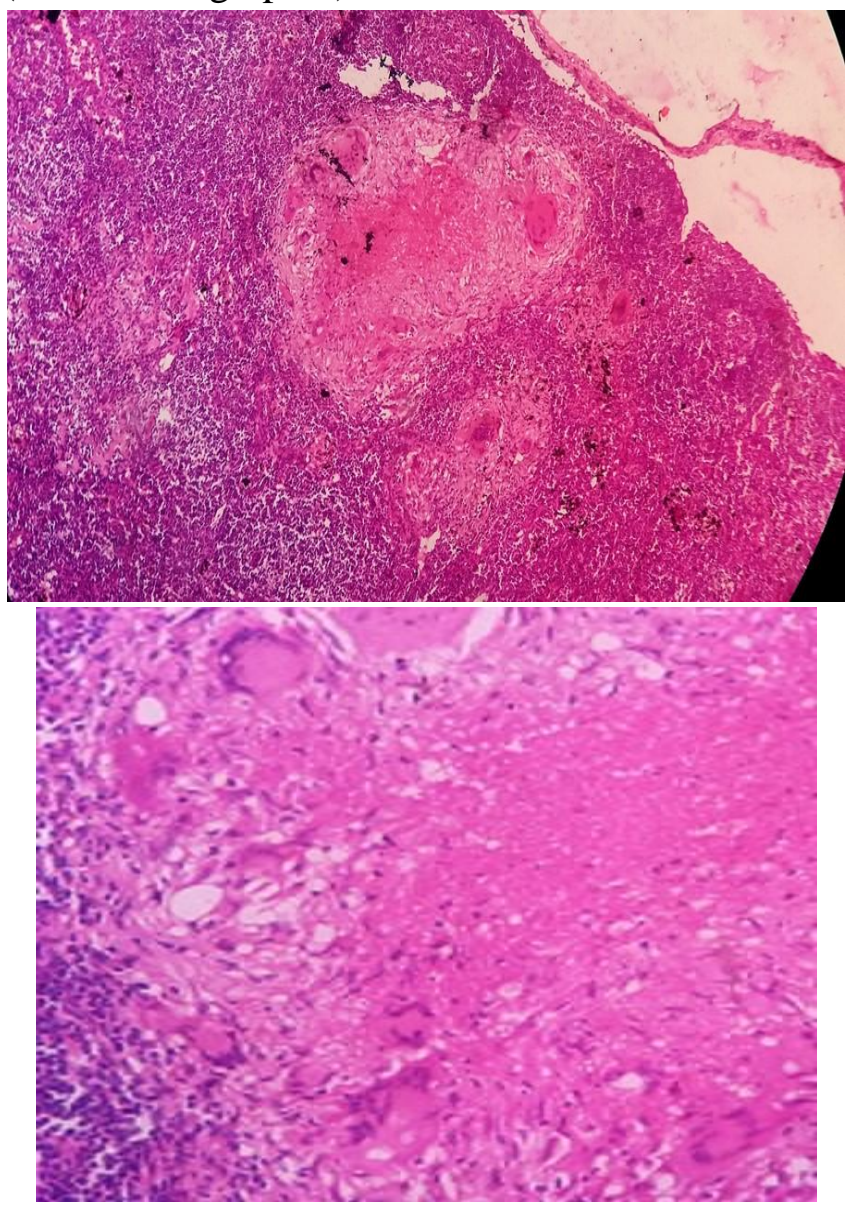

Photomicrograph 4: Microscopic examination showing lymph nodal structure with necrosis and Langhans type giant cell.

\section{DISSCUSSION}

Mature teratomas are the most common histological type of germ cell tumors, followed by seminomas. Germ cell tumors are predominantly found in the gonads, while the anterior mediastinum is the most common extragonadal site $^{(1)}$. Patients present with respiratory distress, chest pain, hemoptysis, cough ${ }^{(1)}$. Teratomas typically range from 2.8 to $3 \mathrm{~cm}$ in diameter, and are cystic and multiloculated but may rarely be predominantly $\quad$ solid $^{(2)}$. Microscopically, 
mesodermal, ectodermal and endodermal elements are seen in varying proportions. Mature elements often take the form of squamous lined cysts. Thymic or pancreatic elements may be seen in mature teratomas. Malignant pulmonary teratomas present as sarcoma or carcinoma with the presence of immature elements like neural tissue ${ }^{(1)}$. Surgical resection is the treatment of choice; and radical extirpation leads to a long recurrence-free survival $^{(3)}$.

Pulmonary hamartoma was first described by Albrecht in $1904^{(4)}$. Hamartoma is a clinically frequent benign lung tumor, accountingfor $77 \%$ of allbenignlung tumors. A classical lesion is nonorganized mixture of epithelial and mesenchymal components such as of fibromyxoid stroma, adipose tissue, cartilage, smooth muscle cells and respiratory epithelium ${ }^{(5)}$.Being more frequent in the sixth decade of life, they are more common in males ${ }^{(6)}$. They are generally asymptomatic and accidentally found in chest radiograph during routine evaluation. Malignant change is practically non-existent; therefore, identification before the definitive management is of utmost importance. These cases need regular follow-up by chest radiograph. In symptomatic cases, large lesions and rapid growth of the lesion surgical interventions are needed that may be limited to enucleation or segmental resections and rarely lobectomy ${ }^{(7)}$

Granulomatous lymphadenitis can be classified into noninfectious and infectious types Infectious granulomatous lymphadenitis can be classified into suppurative lymphadenitis and non suppurative lymphadenitis. Non suppurative lymphadenitis, includes tuberculosis and Bacillus Calmette-Guérin (BCG) lymphadenitis. Tuberculosis is a chronic airborne infectious disease induced by M. tuberculosis. According to WHO TB Statistics global incidence of TB is 9.6 million. Each year 2.2 million people develop TB in India and an estimated 300,000 die from the disease $^{(8)}$. About $90 \%$ of tuberculous lymphadenitis mainly appears in the cervical lymph node and others are in the mediastinal node. The inhaled organism is phagocytized by alveolar macrophages and transported by these cells to hilar lymph nodes. One is formation of epithelioid cell granuloma by CD4+ cells and the other is formation of caseating granuloma by CD8+ cells ${ }^{(9,10)}$. Thereafter, epithelioid granulomas are encapsulated and progress to central caseous necrosis, eventually resulting in healing. The histology of tuberculous lymphadenitis is characterized by central caseous necrosis surrounded by epithelioid cell layer and sporadic Langhans giant cells. Tuberculous lymphadenitis is distinguished from sarcoidosis lymphadenitis by the presence of central caseous necrosis. Organisms are now most easily detected by PCR (11). Finally, healing occurs with calcification.

\section{CONCLUSION}

Biology of teratoma is still laced with controversy. They may be gonadalor extragonadal. Furthermore, they may arise from germ cell following meiosis or may be postmitotic. Some are congenital, a few develop later life from embryonic cell rest. It is very difficult to categorise our case embrologically. Concomitant presence of focal malformation of unorganized mature tissue in the lung does not need any reasoning: but definitely the blending of the above two pathology with a infective granuloma is unique and unearthly in our case.

\section{REFERENCES}

1. Morgan DE, Sanders C, Mcelvein RB. Intrapulmonary teratoma: a case report and review of the literature. J Thorac Imaging. 1992;7:70-77.

2. Moeller KH, Rosado-de-Christenson ML, Templeton PA. Mediastinal mature teratoma: imaging features. AJR Am J Roentgenol. 1997;169:985-990.

3. Takeda S, Miyoshi S, Ohta M, Minami M, et al. Primary germ cell tumors in the mediastinum. A 50-year experience at a single Japanese institution. Cancer. 
2003;97:367-376.

doi:

10.1002/cncr.11068.

4. Jacob S, Mohapatra D, Verghese M. Massivechondroid hamartoma of the lung clinically masquerading as bronchogenic carcinoma.Indian J PatholMicrobiol. 2008 Jan-Mar; 51(1):61-2.

5. Otani Y, Yoshida I, Kawashima O, et al.Benign tumors of the lung: a 20-year surgical experience. Surg Today. 1997; 27(4):310-2.

6. Fine-needle biopsy of hamartomas of the lung.Sinner WNAJR Am J Roentgenol. 1982 Jan; 138(1):65-9.

7. Solitary hamartoma of the lung: is thoracotomy still mandatory?deRooij PD, Meijer S, Calame J, Golding RP, van Mourik JC, StamJNeth J Surg. 1988 Dec; 40(6):145-8.

8. TB India 2016,Revised National Tuberculosis Control Programme Annual Status Report, New Delhi 2016.

9. Marchetti G, Gori A, Catozzi L, Vago L, Nebuloni M, et al.: Evaluation of PCR in detection of Mycobacterium tuberculosis from formalin-fixed, paraffin-embedded tissues : comparison of four amplification assays. J ClinMicrobiol 36:1512-1517, 199874.

10. Polesky A, Grove W, Bhatia G: Peripheral tuberculous lymphadenitis : epidemiology, diagnosis, treatment, and outcome. Medicine 84:350-362, 2005.

11. Samuelson J, von Lichtenberg F: Infectious disease. In : Cotran RS, Kumar V, Robbins SL (eds) : Pathologic basis of disease, 5th ed, Philadelphia, W. B. Saunders, pp.305-377, 1994. 